\title{
Electron Energization Dynamics in Interaction of Self-Generated Magnetic Vortices in Upstream of Collisionless Electron/lon Shocks
}

\section{N. Naseri ( $\square$ nnaseri@utexas.edu )}

The University of Texas

\section{S. G. Bochkarev}

P.N. Lebedev Physics Institute of the Russian Academy of Sciences

\section{Y. Bychenkov}

P.N. Lebedev Physics Institute of the Russian Academy of Sciences

\section{Khudik}

Cornell University

\section{G. Shvets}

Cornell University

\section{Research Article}

Keywords: Electron energization, magnetic vortices, Electron/ion shocks, Relativistic collisionless shocks, supra-thermal particles

Posted Date: November 18th, 2021

DOI: https://doi.org/10.21203/rs.3.rs-1058926/v1

License: (1) This work is licensed under a Creative Commons Attribution 4.0 International License. Read Full License 


\title{
Electron energization dynamics in interaction of self-generated magnetic vortices in upstream of collisionless electron/ion shocks
}

\author{
N. Naseri ${ }^{1,2, *}$, S. G. Bochkarev³ , V. Y. Bychenkov ${ }^{3}$, V. Khudik ${ }^{4}$, and G. Shvets ${ }^{4}$ \\ ${ }^{1}$ Institute for Fusion Studies and Department of Physics, The University of Texas, 1 University Station C1500, Austin, \\ TX, 78712, USA \\ ${ }^{2}$ Physics and Astronomy Department, Middle Tennessee State University, Wiser-Patten Science Hall, 422 Old Main \\ Cir, Murfreesboro, TN 37132, U.S.A \\ ${ }^{3}$ P.N. Lebedev Physics Institute of the Russian Academy of Sciences, Moscow, 119991, Russia \\ ${ }^{4}$ School of Applied and Engineering Physics, Cornell University, Ithaca, New York, 14850, USA \\ *nnaseri@utexas.edu
}

\begin{abstract}
Relativistic collisionless shocks are considered responsible for particle energization mechanisms leading to particle acceleration. While electron energization in shock front region of electron/ion collisionless shocks are the most commonly studied, the mechanism of electron energization in interaction with self-generated magnetic vortices (MVs) in upstream region is still unclear. We investigate electron energization mechanism in upstream region of electron/ion relativistic collisionless shocks, using two dimensional particle-in-cell (PIC) simulations. We discuss mechanism of electron energization which takes place in upstream region of the shock, where the counter stream particles interact with incoming flow. The energy gain of electrons happens during their interaction with evolving fields of self-generated magnetic vortices in this region. Three Fermi-like electron energization scenarios are discussed. Stochastic acceleration of electrons in interaction with fields of MV leads to anisotropic heating of fast electrons due to diffusion in the momentum space of electrons and, finally, synergetic effect of evolving fields of MVs leads to the formation of a power-law tail of supra-thermal particles.
\end{abstract}

\section{Introduction}

Collisionless shocks are unique phenomena in space and astrophysical plasma environment such as supernova remnants, gamma-ray bursts, active galactic nuclei, and binary systems. One of the key features in astrophysical collisionless shocks is particle acceleration. The energized particles either escape the acceleration region and become Cosmic Rays (CR), and/or they interact with ambient backgrounds to produce high energy photons. The spectrum of the radiation emitted by high energy particles from indirect observations and by measurements of CR and gamma-ray bursts (GRBs) spectra show evidences of non-thermal particle acceleration generated by collisionless shocks ${ }^{1-3}$. These observations as well as numerical simulations of unmagnetized relativistic collisionless shocks have shown the evidence of electron energization in shock front along with electron energization in upstream region of the electron/ion shock ${ }^{1,2,4-7}$. While electron energization in shock front region of collisionless shocks are the most commonly studied, electron energization in other large-scale regions of the shocks (e.g. in upstream region) is of great interest. The focus of this study is on electron energization and acceleration mechanism during interaction with self-generated magnetic vortices in upstream of relativistic collisionless electron/ion shocks with no external magnetic field.

Collisionless shocks are believed to be mediated by Weibel instability which leads to fast growth of magnetic field at small scales, plasma isotropization and particle energization at later times ${ }^{8-12}$. A first phase of magnetic field amplification due to Weibel instability happens in the shock front region ${ }^{6,13,14}$. A simultaneous stage of magnetic field growth happens due to development of Weibel instability driven by particles moving ahead of shock front, i.e counter streams, and the the incoming cold plasma streams. Counter stream electron flow are electrons that are either reflected off shock transition region, or escaped interaction with shock transition region. Recently we demonstrated that magnetic vortices (MVs) can self-consistently emerge in the upstream of electron/ion collisionless shocks with no external magnetic field ${ }^{15}$. The early stage of the interaction involves the generation of quasi-linear elongated MVs, which subsequently merge to form circularly shaped MVs (bubble). Localized regions of the strong magnetic field in the form of magnetic dipole vortices (MVs) upstream of the shock were observed in the simulations developed during the nonlinear evolution of the electron and ion filaments.

The magnetic vortex generation occurs at the stage where flow energy transformation into thermal energy takes place ${ }^{15}$. The 
MV's magnetic (and electric) fields grow and their longitudinal size shrink as they move towards shock front after formation. Considerable part of ion kinetic energy is finally converted into the thermal energy (13\%). At the same time, a small group of particles (both electrons and ions) are accelerated to high energies in excess of the initial kinetic energy of flow particles. The electron and ion energy distributions in the vortex domain have considerable nonthermal parts, which confirms the energy dissipation of the bulk ion beam.

Most previous works investigated electron energization in interaction with magnetic structure in shock front region ${ }^{6,7}$. In shock front region, electrons gain energy through Fermi acceleration mechanism ${ }^{16-21}$ by crossing the shock front back and forth. The energy of the electrons increases with time as the number of crossings increases. In this paper, we focus on electron energization processes, which takes place not in the shock front, but in upstream region of the shock, where the counter stream particles interact with incoming flow. The energy gain of electrons happens only during interaction of electrons with evolving fields of MVs. The MVs annihilate before reaching the shock front ${ }^{15}$. We identified three scenarios of Fermi-like electron energization and correspondingly three group of energized electrons in interaction with evolving fields of self generated magnetic vortices in the upstream region of the shock. First and second scenarios correspond to energization of counter stream electrons. These electrons move towards overlapping region, where the cold fresh incoming flows meet the counterstream flows. These electrons can gain significant amount of energy through kick-like stochastic energization process ${ }^{22}$, where electrons receive kicks due to interaction with evolving electric and magnetic fields of the MVs and gain energy during interaction. The third scenario identified in our simulations is energization of incoming electron flow. The incoming electrons can trap in the fields of MVs in the very early stage of their formation. They move with MV towards shock front region and leave MVs in their final stage of MV, while gaining energy in the process of stochastic acceleration in evolving fields of MV.

Tracking a large number of energized electrons from the non-thermal tail of the energy spectrum shows that particles in this region are directly accelerated by the large inductive transverse and longitudinal electric fields of the evolving MVs in this region. The evolution of energy distribution for upstream region shows a power-law tail of supra-thermal particles, which saturates rather quickly after the shock forms and stay stable with power law index of $p \approx 2.1$.

The paper is organized as follows: simulation set-up is the first section, followed by self-generated MVs formation mechanism in upstream, MV structure and non-thermal electron tail formation formation in upstream. Then we present scenarios of electron energization in interaction with self-generated MVs. The last section includes discussion and conclusion.

\section{Results}

\section{Simulation set-up}

The two-dimensional (2D) version of the PIC fully relativistic parallel simulation code VLPL is used ${ }^{23}$. The code was modified to minimize noise properties of numerical instabilities, by using third-order shaped particles and current smoothing. A rectangular simulation box in the $x-z$ plane with the dimensions $L_{x}=1300 l_{p e}$ and $L_{z}=130 l_{p e}$ and the grid sizes $\Delta z=l_{p e} / 10$ and $\Delta x=l_{p e} / 10$ is used. Here $l_{p e}=c / \omega_{p e}$ is the electron inertial length, that is the typical transverse spacial scale of the

filaments, $\omega_{p e}=\sqrt{\frac{4 \pi n_{0} e^{2}}{\gamma_{0} m_{e}}}$ is the electron plasma frequency, $\gamma_{0}$ is the relativistic gamma factor of incoming plasma flow, $e$ and $m$ denote the charge and mass of electron, and $n_{0}$ is the unperturbed density of electrons. Periodic boundary conditions are applied for particles and fields in the transverse $(z)$ direction. The left boundary is reflecting for particles and fields. Simulation stops when counter stream particles reach the right boundary of the simulation domain. Therefore, no particle leaves the domain during simulation. Fresh flow of electrons and ions enter the domain continuously from the right boundary. Each computational cell is initialized with 16 macro-particles: 8 electrons and 8 ions. We assume that initially the electron-ion (e,i) plasma beam with the mass ratio $m_{i} / m_{e}=32$ and equal charges $q_{i}=q_{e}$, equal densities $n_{0}$, and relativistic velocities $v_{x}$ (corresponding to $\gamma_{0} \equiv 1 / \sqrt{1-v_{x}^{2} / c^{2}}=15$ ) moves to the left (in the direction opposite to $x$-axis direction). We chose $m_{i} / m_{e}=32$ as previous studies ${ }^{6}$ showed that for electron-ion mass ratios $m_{i} / m_{e}>30$, properties of the shock do not significantly change with ion mass. Electrons and ions in the incoming plasma beam are assumed to have a negligible energy spread. To reduce the computational effort, the initial contact point of the two counter-propagating streams is modeled as a reflecting wall at $x=0^{6}$. The schematic of the interaction is shown in Fig. 1. After reflecting from the wall (on the left), the reflected and the incoming plasmas stream through each other and form a collisionless shock. The simulation is performed in the reflecting wall frame, where the downstream (thermalized) plasma behind the shock has a vanishing average flow velocity. All densities (electron and ion) and fields (electric and magnetic) are expressed in dimensionless units as $\tilde{N}_{i, e}=n_{i, e} / n_{0}, \tilde{B}_{y}=e B_{y} / m_{e} \omega_{p e} c \sqrt{\gamma_{0}}$, and $\tilde{E}_{x, z}=e E_{x, z} / m_{e} \omega_{p e} c \sqrt{\gamma_{0}}$.

\section{MV formation and structure}

The structure of the fully formed shock at $\omega_{p e} t=1266$ is shown in Fig. 1 . The transversely averaged density $\tilde{N}(x)=\left\langle n(x, z) / n_{0}\right\rangle$ (black line; \langle\rangle denotes transverse averaging over the $z$-dimension) and the color plot of the normalized transverse magnetic field 


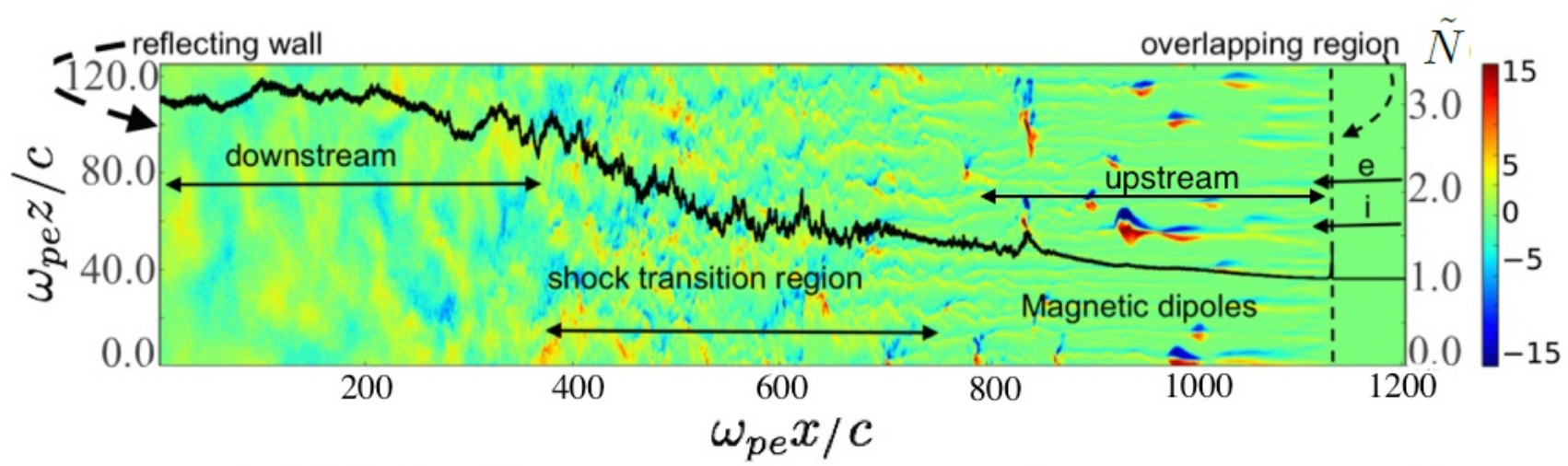

Figure 1. Schematic of the interaction of two interpenetrating electron/ion beams: Color plot of normalized out of plane magnetic field $\left(B_{y}\right)$ in $x-z$ plane at $\omega_{p e} t=1140$. Solid black line (axis on right): transversely averaged density $\tilde{N}$ normalized to upstream unperturbed density $n_{0}$. The dashed line is where the incoming e/i beams meet the counter streams.

$\left(B_{y}\right)$ are plotted in Fig.1 that was chosen to represent a fully developed shock.

Our focus is on upstream region of the shock, where the incoming cold streams interact with isotropized counter stream flow. The upstream region of the relativistic collisionless shocks is dominated by the so called counter stream particles, incoming flow of particles interacting with lower density stream of hot isotropized counter stream electrons and relatively cold counter stream ions, that already escaped the shock region or were never trapped. Here we summarize MV generation and evolution in upstream. In overlapping region, the incoming cold streams of electrons and ions meet with counter stream isotropized electrons and ions with considerable longitudinal and perpendicular velocity spreads (thermal spread). The interaction of counter stream flow with cold incoming flow leads to electron Weibel instability. However, due to thermal spread, the growth rate of electron Weibel instability is low as compared to that for cold beam plasma $\left(\delta_{e} \simeq \sqrt{2} \omega_{p e} v_{x} / c \sim \omega_{p e}\right.$, where $\delta_{e}$ is the growth rate of electron Weibel instability $)^{24}$. Electron Weibel instability initiates quickly as the incoming and counter streams meet in the overlapping region. The growth rate of electron Weibel instability is found to be $0.13 \omega_{p e}$ that is significantly less than for the cold beam plasma case $\delta_{e}=\sqrt{2} \omega_{p e}$, and in agreement with the estimate for a hot electron beam $\simeq \delta_{e}\left(1-\Delta \gamma_{\perp} / \gamma_{0}\right)^{9}$, where $\Delta \gamma_{\perp}$ is the transverse energy spread and $\Delta \gamma_{\perp} \simeq \gamma_{0}$. Initially, small-scale filaments are formed, magnetic field grows and then instability saturates. The maximum value of generated magnetic field is in agreement with estimate for saturation level $B_{y, s} \simeq{\sqrt{\gamma_{0}}}^{25}$. There is no considerable charge imbalance in filaments as electron Weibel instability saturates in overlapping region, where the counter stream flow meet cold flow ${ }^{15}$. At the final stage of electron Weibel instability, the electrons in incoming beam are considerably isotropic. The ion Weibel instability is later developed in the background of well-thermalized electrons. The growth rate of ion Weibel instability is found to be close to $0.34 \omega_{p i}$, (here, $\omega_{p i}=\sqrt{4 \pi e^{2} n_{0} / \gamma_{0} m_{i}}$ is the ion plasma frequency) that is less than the estimate for cold approximation $\left(\delta_{i}=\sqrt{2} \omega_{p i}\right)^{14}$, due to the fact that there is considerable background magnetic field that makes a standard linear analysis not applicable. Current pinching and filament neck formation(see 2(c) below) happens as linear stage of ion Weibel instability terminates, which as mentioned results in magnetic field growth and more pinching which leads to formation of elongated localized magnetic vortices, and their transformation into large-scale magnetic bubbles. Figures 3-(a,b) show the distributions of the out of plane plane magnetic fields $\left(B_{y}\right)$ at the initial (linear) stage of the MV generation at $\omega_{p e} t=1038$ and the saturated nonlinear stage of MV at $\omega_{p e} t=1178$. We typically observed magnetic field enhancement of the MVs by a factor of $\approx 5 \sim \sqrt{m_{i} / m_{e}}$ with respect to the background magnetic field while they propagate towards the shock front ${ }^{15}$. The size of MVs grow to $c / \omega_{p i}$.

Figure 2-(a,b) shows the structure of longitudinal and transverse electric fields $\left(E_{x}, E_{z}\right)$ of nonlinear stage of the MV $\omega_{p e} t=1057$, where the MV is developed but not annihilated yet. Figure 2-(d-f) show the lineouts of the fields and charge density along black overlaid lines shown on top panel. Strong electrostatic electric field is induced around the cavity because of electron evacuation. This field tends to drag the counter stream electrons into the MV. The transverse electric and magnetic fields $\left(E_{z}\right.$ and $\left.B_{y}\right)$ are larger than the longitudinal electrostatic electric field $\left(E_{x}\right)$ as can be seen from Fig. 2-(d-e), however, a combination of both electrostatic and transverse fields determines the entire process of electron energization. The strong Lorentz force $q \mathbf{v} \times \mathbf{B}$, focuses the incoming ion beam, while expelling the incoming electron beam form the center of MV as shown in Fig.2-c. The ion currents are pinched in the self-generated magnetic field. The counter-streaming electron flow follows the ion flow to partly neutralize the beam plasma. However, at the strongly nonlinear stage, significant charge separation appears (see 

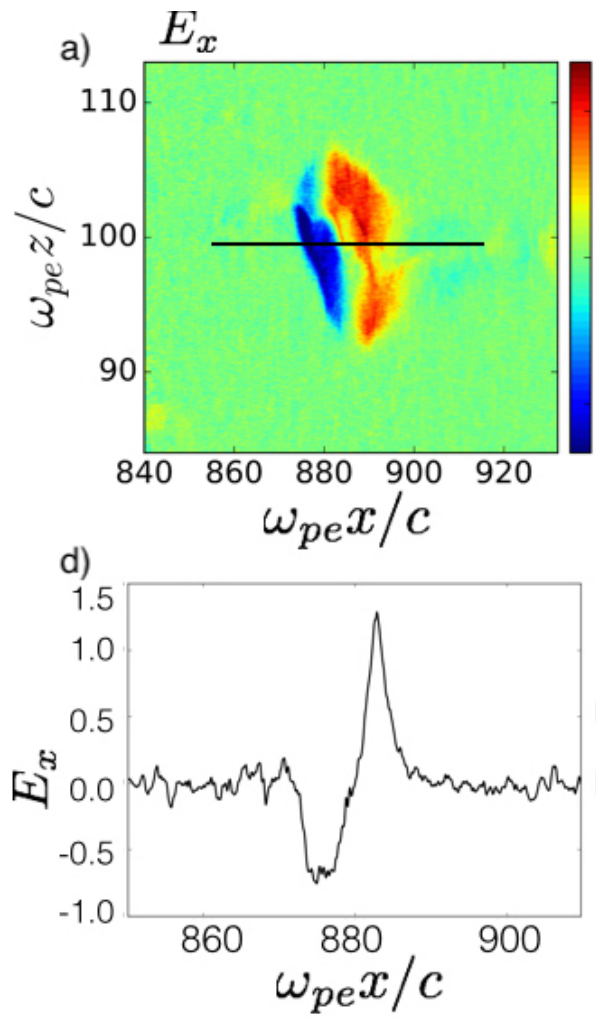

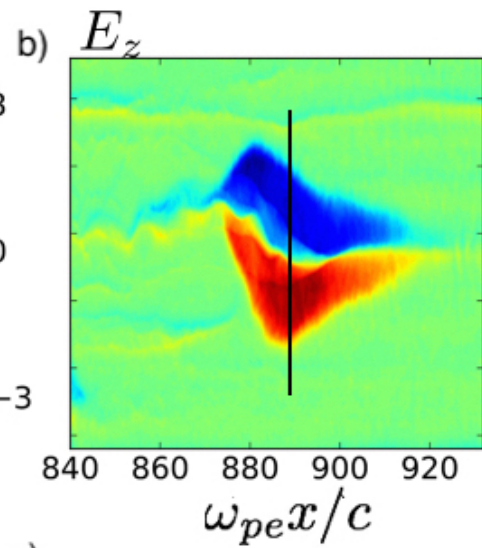

e)

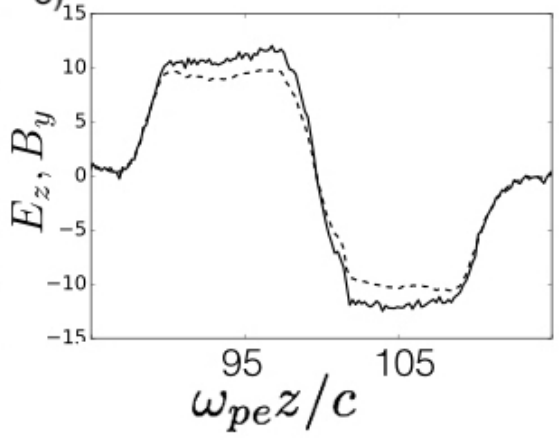

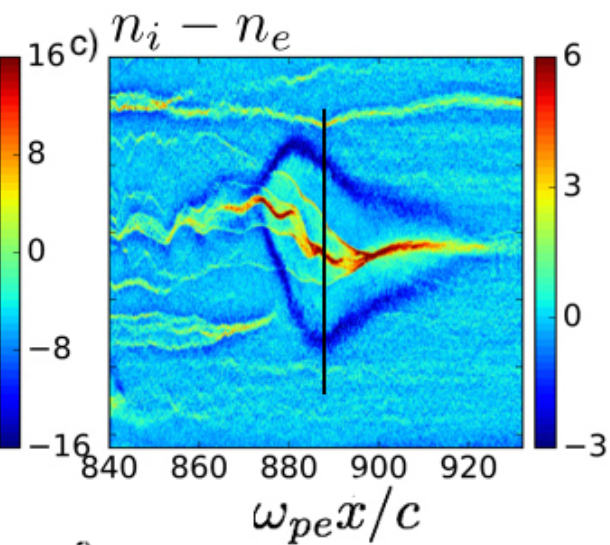

f)

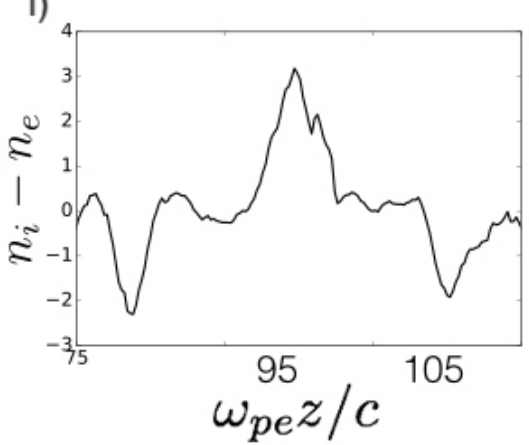

Figure 2. a) Distribution of longitudinal electric field $\left(E_{x}\right)$ of $\left.\mathrm{MV}, \mathrm{b}\right)$ transverse electric field $\left(E_{z}\right)$ c) charge density at $\omega_{p e} t=1057$. d) lineout of the longitudinal electric field along line shown in a). e) lineouts of the transverse electric field (dashed line) and out of plane magnetic field (solid line) corresponding to the line shown in b). f) lineout of charge density along the line shown in c).

charge density in Fig. 2-c). The transverse electric field balances the Lorentz force, $E_{z} \approx v_{x} B_{y} / c$. The ion filament pinching results in an increase in the magnetic field and a consequent increase in the Lorentz force, which can be seen in Fig. 2-(b,c) and corresponding lineouts. The magnetic field of the MV grows during propagation towards downstream. The magnetic field of the MV increases in a non-monotonous manner by a factor of $\approx 5 \sim \sqrt{m_{i} / m_{e}}$ with respect to the background magnetic field ${ }^{15}$. Self-generated MVs move in the plasma density gradient direction toward the shock front ${ }^{26}$. Our simulations show that the axial velocity of the magnetic vortices increases as they move along the density gradient. The drift velocity of magnetic vortices, propagating in a plasma with a density gradient, is estimated to be $v_{A} / c \approx \sqrt{\frac{\alpha B_{y}^{2}}{1+\alpha B_{y}^{2}}}{ }^{27}$, where $\alpha=\frac{m_{e} n_{0}}{m_{i} \gamma_{0} n}$; here, $n$ is the ion density. The longitudinal drift velocity of the magnetic vortices during evolution observed in simulations increases from $\left|v_{x} / c\right| \approx 0.2$ to 0.5 , when the ion density increases from $n_{0}$ to $\approx 1.5 n_{0}$, and $B_{y}$ increases from 3.1 to 15 . Alfvén velocity corresponding to the ion densities and magnetic field amplitudes taken from simulation leads to a value of $v_{A} / c \approx 0.14$ to 0.51 , in agreement with the observed vortex velocities in our simulations. At later times, when dissipation of the vortices begins and their magnetic field degrades, the drift velocity stops growing and starts decreasing, with qualitative agreement with Alfvén velocity formula. This process is periodic, i.e., vortices appear and disappear after time interval of the order of $50 \omega_{p i}^{-1}$.

Figures 3-(c,d) illustrate the electron energy distributions (corresponding to Fig. 3-(a,b), averaged over transverse size of MV along $z$-direction). We can see that the electrons gain a large amount of energy in nonlinear stage of MV. At this time, the transverse electric (and magnetic) field of the MV reaches its maximum. The electron energy has is the largest around the center of the MV and decreases with distance from the center.

The low energy part of the electron energy spectrum is fitted by Maxwell-Jüttner distribution $d N / d \gamma=A \gamma \exp \left(-m_{e} c^{2} \gamma / T_{e}\right)$, where $A$ is the constant, with $T_{e} \approx 2 m_{e} c^{2}$ at $\omega_{p e} t=1038$ and $T_{e} \approx 3 m_{e} c^{2}$ at $\omega_{p e} t=1178$. A non-thermal component with energies up to 200 appears at $\omega_{p e} t=1178$. Figure 3-(f) shows formation of a broad energy distribution that is a superposition of the Maxwell-Jüttner distribution and a plateau, slowly varying energy before fast drop at cutoff. The energy evolution of non-thermal electrons from the tail of spectra averaged over a box enclosing MV, changes with time due to stochastic character of kick-like electron acceleration in electric and magnetic fields of evolving MV (see next section). 

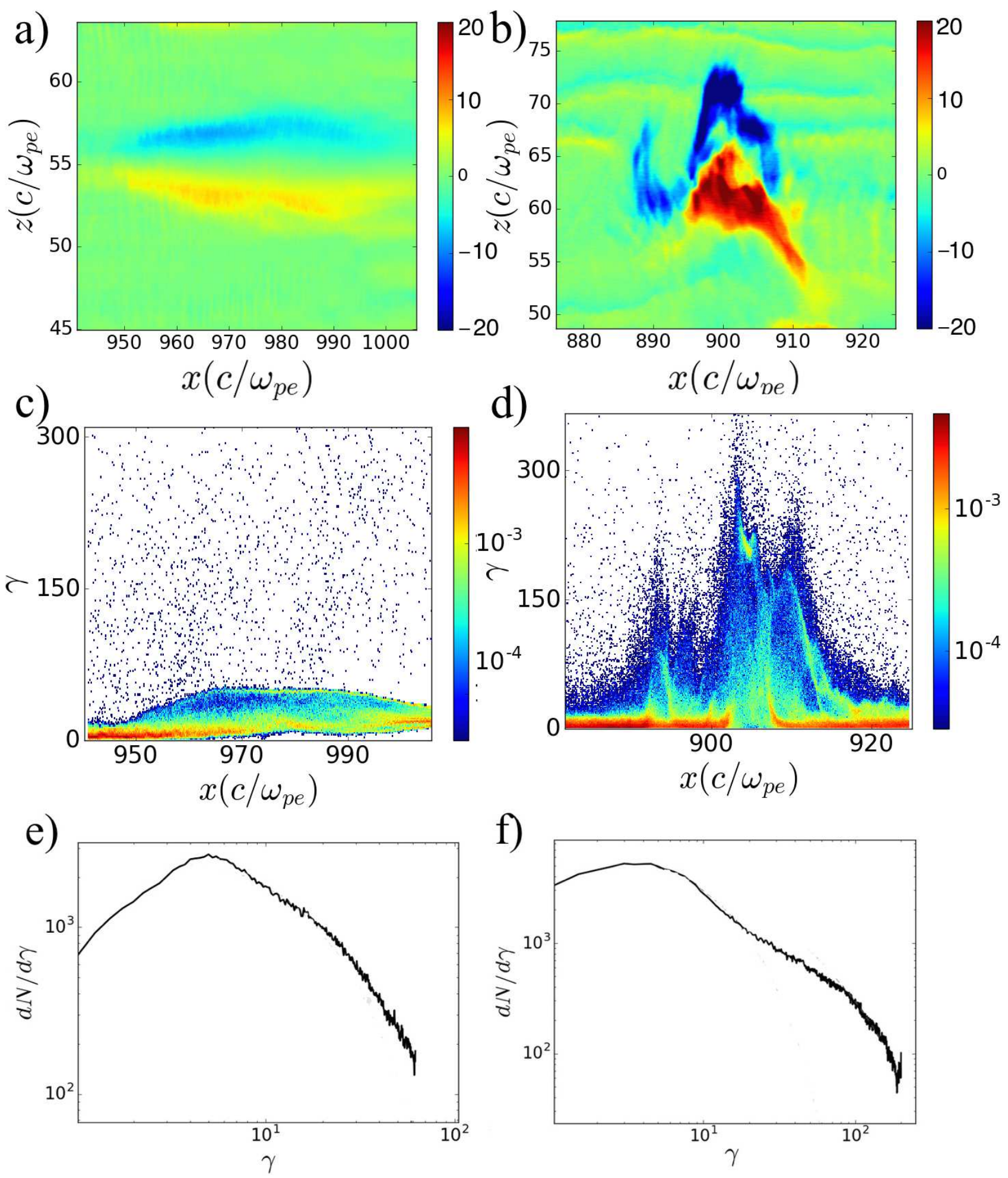

Figure 3. a,b) Magnetic field $\left(B_{y}\right)$ distribution evolution of a typical MV at $\omega_{p e} t=1038$ and 1178. c,d) electron energy distributions averaged over transverse length of MV. e,f) electron energy spectrum evolution corresponding to $\omega_{p e} t=1038$ and 1178 averaged over longitudinal and transverse size of MV. 


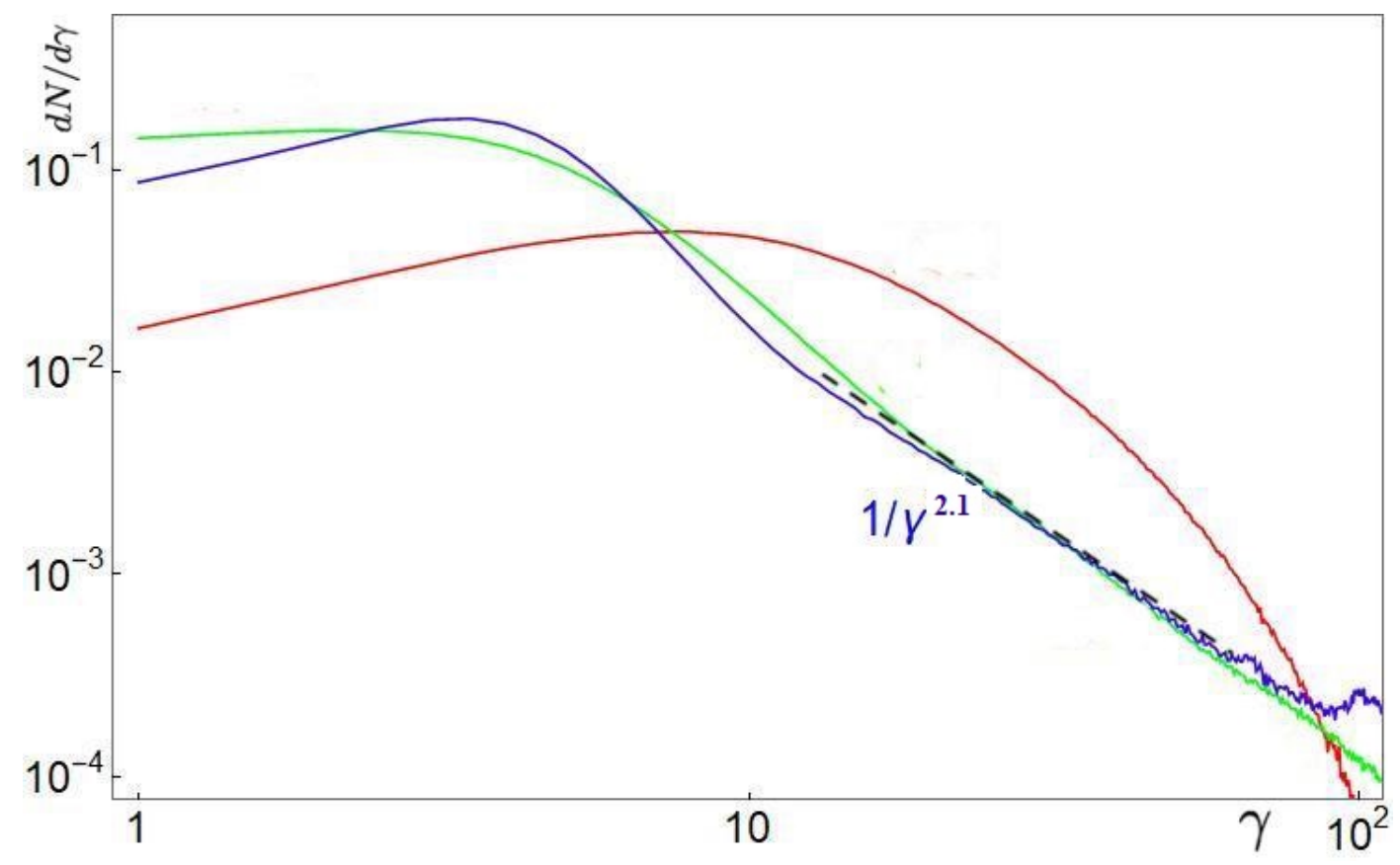

Figure 4. Evolution of energy spectra for whole upstream region for $\omega_{p e} t=162$ (red), 584 (green), 1233 (blue) and corresponding fit (with power index $p \approx 2.1$ ) for $\omega_{p e} t=1233$.

The evolution of energy distribution for upstream region is shown in Fig. 4. MVs begin to appear while the shock is forming $\left(\omega_{p e} t \approx 162\right)$, the longitudinal region where dipoles are located at this time is $\Delta x \approx 36.5 c / \omega_{p e}$. At this time the electrons in this region are thermalized, and the distribution function is close to the Maxwell-Jüttner distribution with $T_{e} \approx 7 m_{e} c^{2}$, no tail formation has yet been observed (Fig. 4- $\left.\omega_{p e} t=162\right)$. After the shock is formed $\left(\omega_{p e} t=420\right)$, the upstream forms and expands, a power-law of supra-thermal particles is formed $\left(d N / \gamma \propto \gamma^{-p}\right)$, which saturates rather quickly by $\omega_{p e} t \approx 584$ and stay stable with power law index of $p \approx 2.1$. Note that shock front is not a part of upstream.

As we mentioned above electron energization (acceleration and heating) in the upstream of relativistic collisionless ion/electron shocks is governed by stochastic diffusion. In the absence of binary collisions its role plays particles interaction with fluctuating EM fields of magnetic vortices. Such effective collisions enable particle acceleration to ultra-relativistic energies vie Fermi-like acceleration mechanism which is similar to acceleration at the front of shock wave, but it results from particle scattering in the interaction process with magnetic fluctuations (self-generated magnetic vortices). This stochastic process of acceleration and heating is characterized by the existence of non-thermal tail of the electron distribution function and considerable part of kinetic energy carried by the non-thermal particles. A power law distribution of accelerated electrons for electron-ion collisionless shock with no external field has been revealed in 2D and 3D PIC simulations with indexes being in the interval range $p=2.1 \div 2.7$ for Fermi ${ }^{6,28,29}$ and Fermi-like acceleration type ${ }^{5,30,31}$. The power law index revealed in the simulation, $p=2.1 \pm 0.1$ (see fit in Fig. 4) for the upstream electron spectrum is consistent with the previous simulations for electron-ions shocks. During acceleration process the magnetic dipoles appear and disappear, at the same time tail is stable for relatively long interval of time ( $\omega_{p e} t>500$ ). Thus, the supra-thermal electron tail is formed due to two processes: (i) as a result of the interaction with magnetic fluctuations (self-generated magnetic vortices) developed at nonlinear stage of filamentation in upstream (main contribution); (ii) as a result diffusion acceleration of electrons by shock front (just a few particles).

\section{Electron energization mechanism}

In the following, we will discuss electron energization scenarios in interaction with magnetic vortices (MVs) resulting in energized electron tail in electron energy distribution in Fig. 3-e and Fig. 4. In order to understand the details of particle acceleration in evolving fields of MV, we tracked the detailed motion of the electrons from the tail of the energy spectrum. The work done by each component of electric field on each particle is calculated throughout the simulation: $W_{i}=\int_{0}^{t} d t^{\prime}\left(p_{i} / \gamma m_{e, i}\right)\left( \pm e E_{i}\right)$, where $i=x, y, z$. Three distinct types of energized electrons were observed in interaction of electrons with MV. All groups of electrons show stochastic behavior of trajectories in the MV fields. Our analysis of many MVs in the upstream of the shock indicates that $\sim 90 \%$ of the energized electron flow from non thermal tail of energy spectrum move towards (or return) to the 

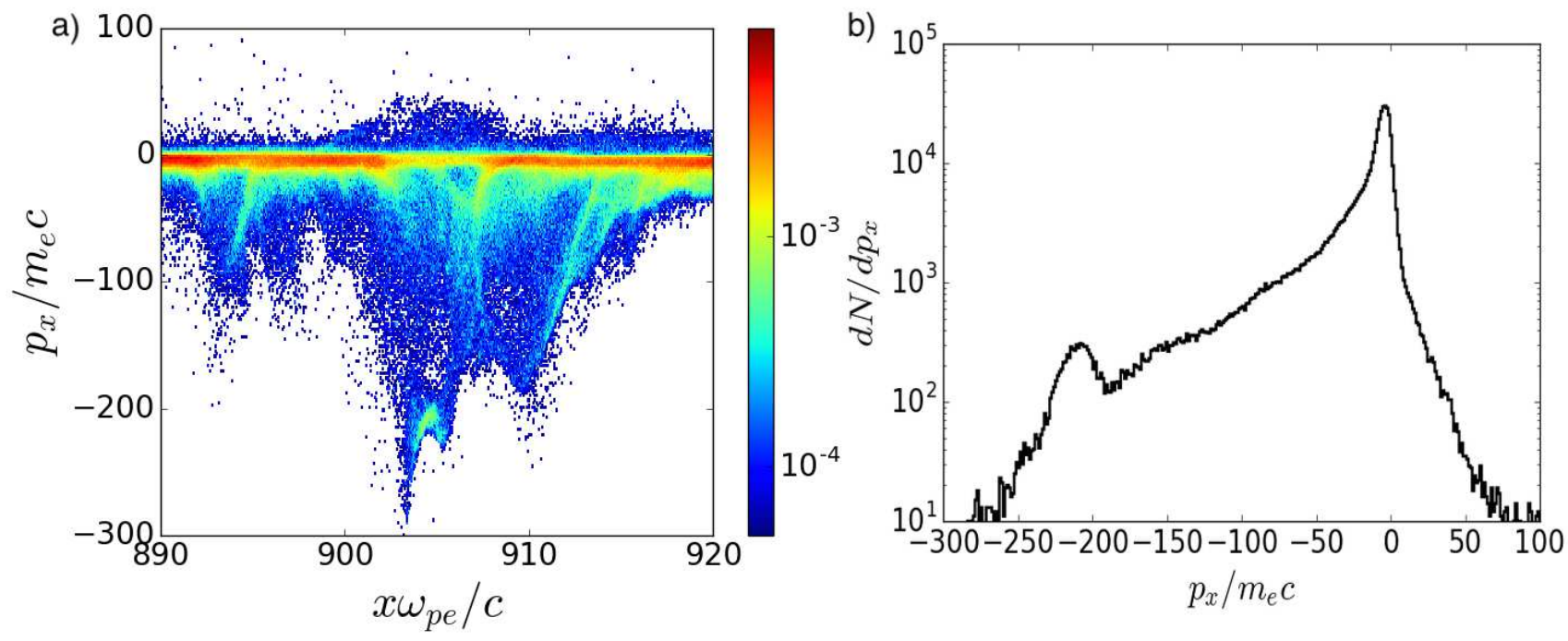

Figure 5. a) electron momentum distribution $p_{x} / m_{e} c$ along $x$-direction averaged over transverse size of the MV. b) electron phase spectrum corresponding to a) at $\omega_{p e} t=1178$.

shock region after gaining energy from electric fields of MV. Figure 5 shows the electron phase distribution averaged over transverse size of the MV at $\omega_{p e} t=1178$ (see Fig. 3-(d,e)). Energetic electrons from the tail of the energy spectrum have negative longitudinal momentum. The longitudinal momentum distribution of the electrons (Fig. 5) averaged over the box shown in Fig. 3-b, illustrates the population of electrons moving towards (or returning) to the shock transition region. The peak around $p_{x} \sim-220 m_{e} c$, shown in Fig. 5-b corresponds to the population of electrons shown in Figs. 5-a, 3-d. A few percent of counter stream electrons from the non-thermal tail of energy spectrum are pre-accelerated before interaction with MV. These electrons gain some extra energy and continue towards upstream after leaving MV. The third type of energetic electrons are from the incoming electron flow. These electrons trap in linear stage of MV formation and move with MV until annihilation. They gain energy from the electric fields of MV and leave MV while move towards shock transition region.

We start with the first type of electron energization mechanism: a typical counter stream electron moving along $+x$ direction, towards upstream, experiences the magnetic force of $e v_{z} B_{y}(-\hat{\mathbf{x}})\left(v_{z}<0, B_{y}>0\right.$ in this case (Fig.6-(a,b,c,d) )) along $-x$-direction which is larger than the electric force $-e E_{x}$ (note that $\left|E_{x}\right|<\left|E_{z}\right|,\left|B_{y}\right|$, See Fig. 2 and supplementary material 1). Supplementary material 1 clearly shows the kick-like energization scenario for such electrons during their interaction with nonlinear MV. This causes the electron to abruptly turn and move in the opposite direction towards shock transition region. Meanwhile it gains the energy while moving in the positive lobe of the longitudinal electric field of MV during reflection. The energy gain of electron from longitudinal electric field continues during reflection of the electron $\left(-e E_{x} d x>0,\left(E_{x}>0, d x<0\right)\right)$. At the same time the electron gains energy as it moves in transverse electric field $\left(-e E_{z} d z, E_{z}>0, d z<0\right.$ Supplementary material 1$)$, and therefore the energy of the electron increases significantly. As the electron passes the center of the MV and moves towards the negative lobe of longitudinal electric field of the dipole, it loses a fraction of its energy and finally, the electron leaves the MV at its final stage with energy gain of more than an order of magnitude larger than its original energy and moves towards the shock region. A typical trajectory of such energetic electron from the tail of the energy spectrum is illustrated in Fig. 6-a overlaid on transverse electric field distribution at $\omega_{p e} t=1132$ showing the return of the electron towards shock region. Figure 6-b shows the energy gain of the electron plotted as a function of $x$, showing the energy gain and return of electron during this process. The evolution of total energy and work done by the electric field components is plotted in Fig. 6-c. The work done by by both longitudinal and transverse electric field of the MV is leading to the energization of such electrons. Figure 6 shows energetic electron behavior from tail of the energy spectrum corresponding to energization mechanism discussed here. Figure 6-a shows the energy gain of such electrons as a function of longitudinal direction $(x)$, showing the return of these electrons while gaining a large amount of energy. We can see that electrons interacting with nonlinear stage of MV, where fluctuating fields reach their largest magnitude, gain more energy than the electrons interacting with MV at earlier times, when the fields are still growing. Figure 6- $(b, c)$ show the energy gain time evolution and longitudinal momentum of such particles, confirming their return to shock region. A characteristic behavior of these energetic electrons is that they return to the shock region after interaction with MV. 

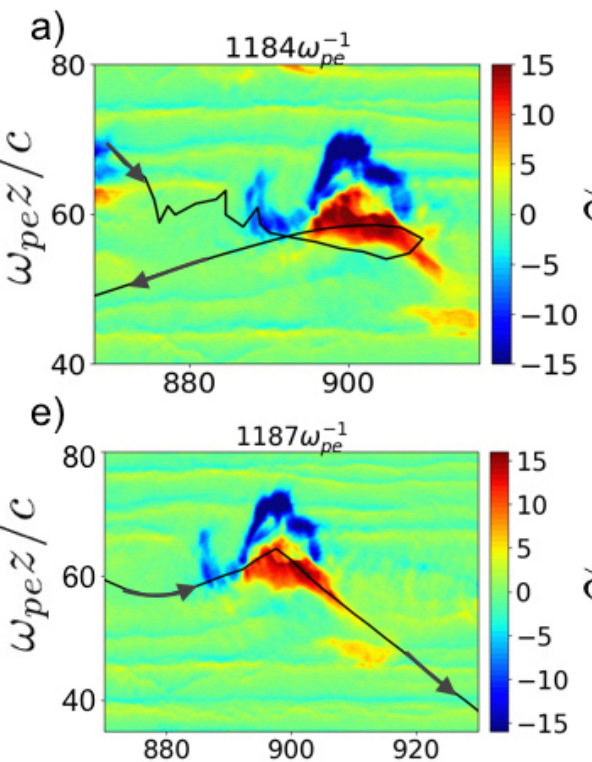

i)

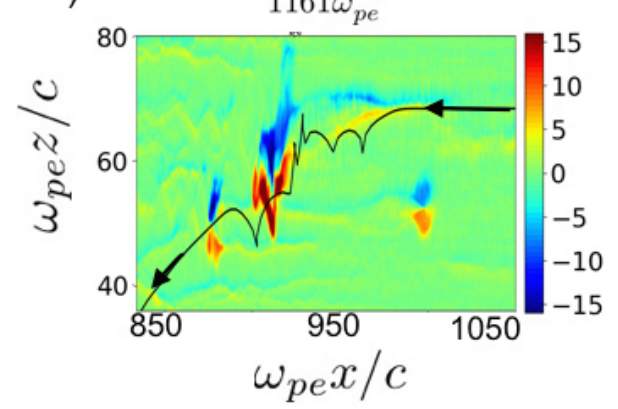

b)

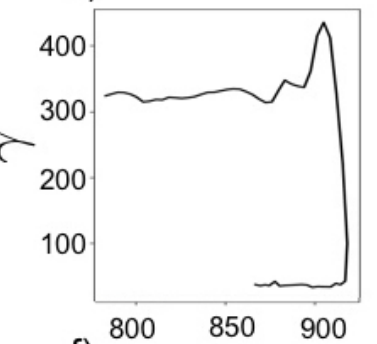

f)

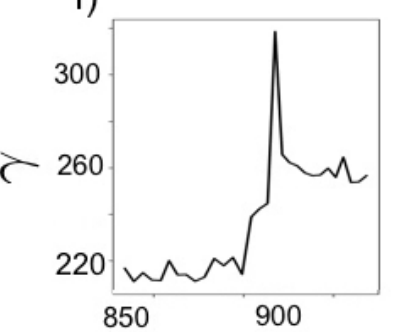

j)

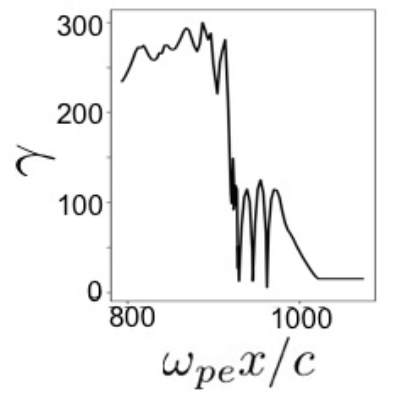

c)

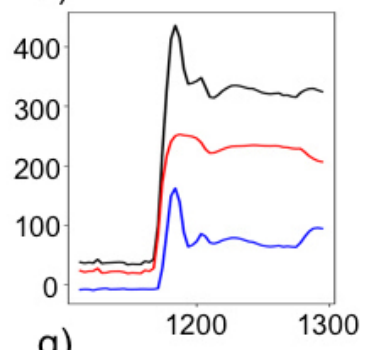

g)

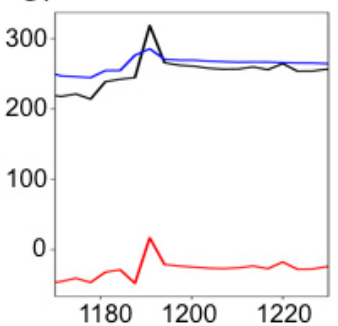

k)

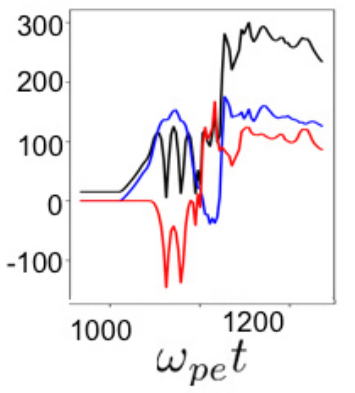

d)

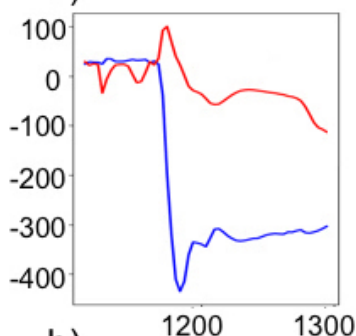

h)

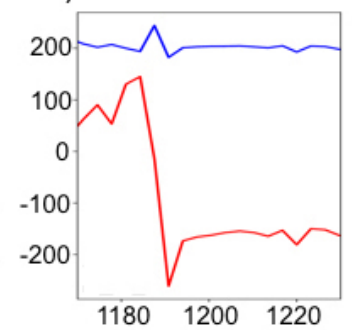

I)

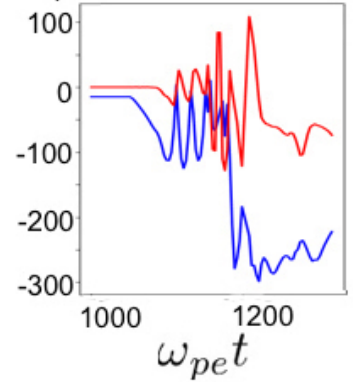

Figure 6. Left column (a, e, i) shows the distributions of transverse electric field of MV. The trajectories of energized electrons are superimposed and shown by black color. The middle column $(\mathrm{b}, \mathrm{f}, \mathrm{j})$ shows the electron energy as a function of $x$. The third column (c, g, k) illustrates electron energies (black) and work done by longitudinal (blue) and transverse (red) electric fields on the particle as a function of time. The fourth column (d, h, i) shows the longitudinal (blue) and transverse (red) momentum $\left(p_{x} / m c, p_{z} / m c\right)$ of electrons as a function of time. 
The second type of energized electrons are the pre-accelerated counter stream electrons moving toward upstream of the shock. These electrons already have large energies $\left(\gamma_{\text {initial }}>160\right.$ for the typical electron in Fig. 6-e) moving towards upstream prior interacting with nonlinear MV. The transverse component of magnetic Lorentz force $\left(-e v_{x} B_{y}(\hat{\mathbf{z}})\right)$ kicks the electron out of MV. For the typical electron shown in Fig. 6-e $v_{x}>0, B_{y}>0$, therefore the magnetic Lorentz force is along $-\hat{\mathbf{z}}$ and the electron is kicked out of MV (see Supplementary material 2). The electron loses energy while moving into MV $\left(E_{z}>0, d z>0\right)$ (Fig. 6-e). Then the magnetic force of MV divert the electron, and the electron gains energy while moving out of $\mathrm{MV}\left(E_{z}>0, d z<0\right)$. It then continues towards upstream. Figure 6-e shows the trajectory of such electron overlaid on electric field distribution at $\omega_{p e} t=1187$, showing typical electron continue towards upstream after interaction with fluctuating fields of MV at its nonlinear stage . Figure 6-f shows the electron energy as a function of $x$ which shows some energy gain for electron before leaving MV. Figure 6-g shows that most of the energy gain is from transverse electric field.

The third type of energetic electrons from the tail of the energy spectrum are the incoming electron flow. The incoming electron enters upstream of the shock and quickly traps in the electric field of MV during linear stage of MV formation. The longitudinal electric field of MV traps the electron, so the electron moves with MV towards shock transition region. At the same time, fluctuating electric and magnetic fields of MV grow significantly. The trapped electron reflects from one lobe to the other due to transverse magnetic Lorentz force. Its energy oscillates rapidly between 15 and $100 m_{e} c^{2}$ for typical electron shown in Fig. 6-i (Supplementary material 3). Finally at the final stage of MV, the electron leaves MV while gaining energy mostly from transverse electric field and continues towards shock transition region (Fig. 6-i).

We never observed incoming electrons returning to upstream for obvious reason: the incoming electron flow has mostly longitudinal momentum, therefore the longitudinal force of $-e v_{z} B_{y} \hat{\mathbf{x}}$ is not large enough to return the electron to upstream (because $v_{z}$ is very small or zero). In addition, the longitudinal electric field force of $-e E_{x} \hat{\mathbf{x}}$ helps electrons to trap in the MV and move with MV.

\section{Discussion}

We studied the process of electron energization in interaction with MVs in the upstream of electron/ion shock using 2D PIC simulations. MVs are self-consistently generated in upstream of electron/ion collisionless shock ${ }^{15}$. The fields of MVs grow as they move toward shock region. Electron energization happens as the electrons (counter-stream and incoming flow) interact with electric fields of MVs in upstream. Three Fermi-like scenarios of stochastic electron energization were discussed: Large fields of MVs can return counter stream electrons towards shock, meanwhile electrons gain significant amount of energy during interaction with fluctuating electric fields of MV. The second energization mechanism happens when counter stream electrons receive a kick during interaction with MVs due to Lorentz force. The electrons gain energy while being kicked (kick-acceleration). The energized electrons continue moving towards overlapping region. The third scenario is energization of incoming cold flow of electrons. Incoming electron flow can trap in the electric field of the MV in its linear stage. The electrons move with MV as fields of MV grow and finally leave MV while gaining energy as MV annihilates. These electron energization processes happen on time scale of MV formation and evolution and does not require long times as it is needed in shock front. Stochastic acceleration results in generation of non-thermal particles that form a power law spectrum. The energy of non-thermal electrons from the tail of spectrum changes with time due to stochastic character of Fermi-like electron acceleration in electric and magnetic fields of evolving MV. After the shock is formed, the upstream forms and expands, a power-law of supra-thermal particles is formed, which saturates rather quickly by $t \omega_{p e}=600$ and stay stable with power law index of $p \approx 2.1$.

In reality, magnetic vortices are three dimensional entities, where the current flow will be along its central axis and electron current flows around the cavity and forms a spheroidal or ellipsoidal shell. It is expected that magnetic vortices would be self-generated in upstream of electron/ion shocks. However, due to the additional degree of freedom, MV life time might be shorter than the 2D calculations which can potentially change accelerated electron population in interaction with the MVs.

It is worth mentioning that high power laser facilities provide a unique opportunity for laboratory experiments using plasma flows driven by high energy laser systems which opens a new era in astrophysics and space exploration. Laboratory experiments open the door to investigate the electron-ion sub-relativistic and relativistic collisionless shocks, magnetic field generation and amplification, magnetic reconnection, and particle acceleration in a short temporal and limited spatial scale via laser-plasma interactions ${ }^{32-34}$. Energy transfer from fast ion flow to electromagnetic fields and fast particles (electrons and ions), at a time scale much shorter than electron-ion collisional energy exchange time can be modelled in laboratory conditions. Simulations are scalable to astrophysical conditions with the plasma densities of the order of a few particles per $\mathrm{cm}^{3}$ to the laboratory scale with laser-produced plasmas with densities about 18-20 orders of magnitude higher ${ }^{35}$. Reaching the collisionless regime also allows the instability dynamics to be described by dimensionless parameters and scaled between laboratory and astrophysical systems. 


\section{Methods}

The two-dimensional (2D) version of the PIC fully relativistic parallel simulation code VLPL is used ${ }^{23}$. The code was modified to minimize noise properties of numerical instabilities, by using third-order shaped particles and current smoothing. A rectangular simulation box in the $x-z$ plane with the dimensions $L_{x}=1300 l_{p e}$ and $L_{z}=130 l_{p e}$ and the grid sizes $\Delta z=l_{p e} / 10$ and $\Delta x=l_{p e} / 10$ is used. Here $l_{p e}=c / \omega_{p e}$ is the electron inertial length, that is the typical transverse spacial scale of the

filaments, $\omega_{p e}=\sqrt{\frac{4 \pi n_{0} e^{2}}{\gamma_{0} m_{e}}}$ is the electron plasma frequency, $\gamma_{0}$ is the relativistic gamma factor of incoming plasma flow, $e$ and $m$ denote the charge and mass of electron, and $n_{0}$ is the unperturbed density of electrons. Periodic boundary conditions are applied for particles and fields in the transverse $(z)$ direction. The left boundary is reflecting for particles and fields. Simulation stops when counterstream particles reach the right boundary of the simulation domain. Therefore, no particle leaves the domain during simulation. Fresh flow of electrons and ions enter the domain continuously from the right boundary. Each computational cell is initialized with 16 macro-particles: 8 electrons and 8 ions. We assume that initially the electron-ion (e,i) plasma beam with the mass ratio $m_{i} / m_{e}=32$ and equal charges $q_{i}=q_{e}$, equal densities $n_{0}$, and relativistic velocities $v_{x}$ (corresponding to $\gamma_{0} \equiv 1 / \sqrt{1-v_{x}^{2} / c^{2}}=15$ ) moves to the left (in the direction opposite to $x$-axis direction). To reduce the computational effort, the initial contact point of the two counter-propagating streams is modeled as a reflecting wall at $x=0^{6}$. The simulation is performed in the reflecting wall frame, where the downstream (thermalized) plasma behind the shock has a vanishing average flow velocity. All densities (electron and ion) and fields (electric and magnetic) are expressed in dimensionless units as $\tilde{N}_{i, e}=n_{i, e} / n_{0}, \tilde{B}_{y}=e B_{y} / m_{e} \omega_{p e} c \sqrt{\gamma_{0}}$, and $\tilde{E}_{x, z}=e E_{x, z} / m_{e} \omega_{p e} c \sqrt{\gamma_{0}}$.

\section{References}

1. Piran, T. The physics of gamma-ray bursts. Rev. Mod. Phys. 76, 1143-1210, DOI: https://doi.org/10.1103/RevModPhys. 76.1143 (2004).

2. Gehrels, P., N. Meszars. Gamma-ray bursts. Science 337, 932-936, DOI: 10.1126/science.1216793 (2012).

3. Warren, J. S. et al. Cosmic-ray acceleration at the forward shock in tycho's supernova remnant: Evidence from chandra x-ray observations. Astrophys. J. 634, 376-389, DOI: 10.1086/496941 (2005).

4. Panaitescu, A. \& Kumar, P. Properties of relativistic jets in gamma-ray burst afterglows 1 warning. Astrophys. J. 571, 779-789, DOI: 10.1086/340094 (2002).

5. Hededal, C. B., Haugbolle, T., Frederiksen, J. T. \& Nordlund. Non-fermi power-law acceleration in astrophysical plasma shocks. Astrophys. J. L107, 617-620, DOI: 10.1086/427387 (2004).

6. Spitkovsky, A. On the structure of relativistic collisionless shocks in electron-ion plasmas. Astrophys. J. 637, L39-L42, DOI: $10.1086 / 527374(2008)$.

7. Ardaneh, K., Cai, D. \& Nishikawa, K. Collisionless electron-ion shocks in relativistic unmagnetized jet-ambient interactions: Non-thermal electron injection by double layer. Astrophys. J. 827, 124-139, DOI: https://doi.org/10.3847/ 0004-637X/827/2/124 (2016).

8. Weibel, E. Spontaneously growing transverse waves in a plasma due to an anisotropic velocity distribution. Physicsl Rev. Lett. 2, 83-84, DOI: https://doi.org/10.1103/PhysRevLett.2.83 (1959).

9. V., M. M. \& Loeb, A. Generation of magnetic fields in the relativistic shock of gamma-ray burst sources. Astrophys. $J$. 526, 697-706, DOI: https://doi.org/10.1086/308038 (1999).

10. Silva, L. O., Fonseca, R. A., Tonge, J. W., Dawson, W. B., J. M. Mori \& Medvedev, M. V. Generation of magnetic fields in the relativistic shock of gamma-ray burst sources. Astrophys. J. 526, 697-706, DOI: https://doi.org/10.1086/308038 (2003).

11. Medvedev, M., Fiore, M., Fonseca, R. A., Silva, L. O. \& Mori, W. B. Long-time evolution of magnetic fields in relativistic gamma-ray burst shocks. Astrophys. J. 618, L75-L78, DOI: https://doi.org/10.1086/427921 (2005).

12. Nishikawa, K., Hardee, P. E., Hededal, C. B. \& Fishman, G. J. Particle acceleration, magnetic field generation, and emission in relativistic shocks. Astrophys. J. 38, 1316-1319, DOI: https://doi.org/10.1016/j.asr.2005.01.036 (2006).

13. Bret, A. Weibel, two-stream, filamentation, oblique, bell, buneman...which one grows faster? Astrophys. J. 699, 990-1003, DOI: https://doi.org/10.1088/0004-637X/699/2/990 (2009).

14. Stockem Novo, A., Bret, A., Fonseca, R. A. \& Silva, L. O. Shock formation in electron-ion plasmas: Mechanism and timing. Astrophys. J. 803, L29-L34, DOI: https://doi.org/10.1088/2041-8205/803/2/L29 (2015).

15. Naseri, N. et al. Growth and propagation of self-generated magnetic dipole vortices in collisionless shocks produced by interpenetrating plasmas. Phys. Plasmas 25, 012118, DOI: https://doi.org/10.1063/1.5008278 (2018). 
16. Fermi, E. On the origin of the cosmic radiation. Phys. Rev. 75, 1169, DOI: https://doi.org/10.1103/PhysRev.75.1169 (1949).

17. Bell, A. R. The acceleration of cosmic rays in shock fronts - i. Mon. Notices Royal Astron. Soc. 182, 147-156, DOI: https://doi.org/10.1093/mnras/182.2.147 (1978).

18. Bell, A. R. Cosmic ray acceleration. Astropart. Phys. 43, 56-70, DOI: https://doi.org/10.1016/j.astropartphys.2012.05.022 (2013).

19. Blandford, R. D. \& Ostriker, R. D. Particle acceleration by astrophysical shocks. Astrophys. J. 221, L29-L32, DOI: https://doi.org/10.1086/182658 (1978).

20. Drury, L. O. An introduction to the theory of diffusive shock acceleration of energetic particles in tenuous plasmas. Reports on Prog. Phys. 46, 973-1027, DOI: https://doi.org/10.1088/0034-4885/46/8/002 (1983).

21. Blandford, R. \& Eichler, D. Particle acceleration at astrophysical shocks: A theory of cosmic ray origin. Phys. Reports 154, 1-75, DOI: https://doi.org/10.1016/0370-1573(87)90134-7 (1987).

22. Bochkarev, S., d'Humières, E., Korneev, P., Bychenkov, V. \& Tikhonchuk, V. The role of electron heating in electromagnetic collisionless shock formation. High Energy Density Phys. 17A, 175, DOI: https://10.1016/j.hedp.2015.01.001 (2015).

23. Pukhov, A. Three-dimensional electromagnetic relativistic particle-in-cell code vlpl (virtual laser plasma lab). J. Plasma Phys. 61, 425 - 433, DOI: https://doi.org/10.1017/S0022377899007515 (1999).

24. Califano, F., Prandi, R., Pegoraro, F. \& Bulanov, S. V. Kinetic saturation of the weibel instability in a collisionless plasma ). Phys. Rev. E 57, 7048, DOI: https://doi.org/10.1103/PhysRevE.57.7048 (1998).

25. Bret, A. et al. Collisionless shock formation, spontaneous electromagnetic fluctuations, and streaming instabilities plasma ). Phys. Plasmas 20, 042102, DOI: https://doi.org/10.1063/1.4798541 (2013).

26. Yadav, S. \& Das, A. Nonlinear studies of fast electron current pulse propagation in a two dimensional inhomogeneous plasma ). Phys. Plasmas 17, 052306, DOI: https://doi.org/10.1063/1.3407621 (2010).

27. Gedalin, M. Linear waves in relativistic anisotropic magnetohydrodynamics ). Phys. Rev. E 47, 4354, DOI: https: //doi.org/10.1103/PhysRevE.47.4354 (1993).

28. Eichler, D. \& Waxman, E. The efficiency of electron acceleration in collisionless shocks and gamma-ray burst energetics ). Astrophys. J. 627, 861-867, DOI: https://doi.org/10.1086/430596 (2005).

29. Martins, S. F., Fonseca, R. A., Silva, L. O. \& Mori, W. B. Ion dynamics and acceleration in relativistic shocks ). Astrophys. J. 695, L189-L193, DOI: https://doi:10.1088/0004-637X/695/2/L189 (2009).

30. Trubnikov, B. On the possible generation of cosmic rays in plasma pinches ). Physics-Uspekhi 33, 1061-1071, DOI: https://doi.org/10.3367/UFNr.0160.199012g.0167 (1990).

31. Haugbølle, T. Three-dimensional modelling of relativistic collisionless ion-electron shocks ). The Astrophys. J. Lett. 739, L42, DOI: https://doi:10.1088/2041-8205/739/2/L42 (2011).

32. Fiuza, F. \& et al. Electron acceleration in laboratory-produced turbulent collisionless shocks. Nat. Phys. 16, 916, DOI: https://doi:10.1038/s41567-020-0919-4 (2020).

33. Huntington, C. \& et al. Observation of magnetic field generation via the weibel instability in interpenetrating plasma flows. Nat. Phys. 11, 173, DOI: https://doi:10.1038/NPHYS3178 (2015).

34. Burdonov, K. \& et al. Inferring possible magnetic field strength of accreting inflows in exor-type objects from scaled laboratory experiments. Astron. Astrophys. 648, A81, DOI: https://10.1051/0004-6361/202040036 (2021).

35. Davis, S., Capdessus, R., D’Humières, E. E. \& Tikhonchuk, V. Numerical simulations of energy transfer in counterstreaming plasmas. High Energy Density Phys. 9, 231, DOI: https://doi:10.1016/j.hedp.2012.11.009 (2013).

\section{Acknowledgements}

This work was supported by the US Department of Energy under a Grant No. DE-NA0003879. The authors also wish to thank Texas Advanced Computing System for providing computational resources and their help to restore our simulation data.

\section{Author contributions statement}

N. Naseri performed simulations, analyzed results, generated figures and prepared manuscript. N. Naseri, V. Yu. Bochkarev and S. G. Bochkarev discussed the results. All authors reviewed the manuscript. 


\section{Additional information}

Correspondence and requests for materials should be addressed to N. Naseri. 


\section{Supplementary Files}

This is a list of supplementary files associated with this preprint. Click to download.

- LegendsforSupplementaryVideos.pdf

- track76.mov

- track70.mov

- track74.mov 